la revue La revue pour l'histoire du CNRS

POUR L'HISTOIRE DU CNRS

$7 \mid 2002$

La biologie | Menaces sur les sciences sociales vers

1980

Natures, Sciences et Sociétés. «Sciences pour l'ingénierie de l'environnement »

Édition spéciale, vol. 10, sup. 1. Elsevier, avril-juin 2002

Girolamo Ramunni

OpenEdition

Journals

Édition électronique

URL : https://journals.openedition.org/histoire-cnrs/406

DOI : 10.4000/histoire-cnrs.406

ISSN : 1955-2408

Éditeur

CNRS Éditions

Édition imprimée

Date de publication : 5 novembre 2002

ISBN : 978-2-271-06067-9

ISSN : 1298-9800

Référence électronique

Girolamo Ramunni, « Natures, Sciences et Sociétés. « Sciences pour l'ingénierie de

l'environnement »», La revue pour l'histoire du CNRS [En ligne], 7 | 2002, mis en ligne le 07 mars 2006,

consulté le 20 mai 2021. URL : http://journals.openedition.org/histoire-cnrs/406 ; DOI : https://doi.org/ 10.4000/histoire-cnrs.406

Ce document a été généré automatiquement le 20 mai 2021

Comité pour l'histoire du CNRS 


\title{
Natures, Sciences et Sociétés. "Sciences pour l'ingénierie de l'environnement »
}

\author{
Édition spéciale, vol. 10, sup. 1. Elsevier, avril-juin 2002
}

\section{Girolamo Ramunni}

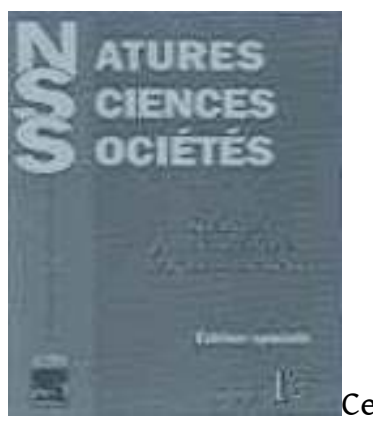

Ce numéro spécial contient, outre des articles sur les recherches menées au Cemagref en modélisation, des articles sur la place des modèles dans la recherche actuelle. Le recours aux modèles dans la recherche actuelle. Le recours aux modèles, si on prend ce terme au sens large, a précédé la révolution scientifique et a accompagné le développement de nombreuses disciplines. Il n'est pas étonnant dès lors que la physique soit le premier domaine à s'être servi des modèles. Aujourd'hui, de l'économie à la physique, de la biologie à la sociologie, les modèles semblent être devenus l'une des méthodes qui dépassent les barrières disciplinaires et constituent une sorte d'unité dans la diversité des champs disciplinaires.

1 Ce numéro spécial de NSS est consacré à la modélisation dans le domaine de l'environnement. Outre l'actualité de la thématique, un point est à souligner: l'environnement est un champ de recherche ou l'interdisciplinarité est la contrainte majeure. La modélisation peut être présentée comme la méthodologie permettant de favoriser la pratique de l'interdisciplinarité.

2 L'article de Claudine Schmidt-Lainé et Alain Pavé présente la diversité des méthodes de modélisation. Ce qui est mis en valeur est le rôle des modèles, passages indispensables 
pour comprendre la dynamique de l'environnement, l'ingénierie de l'environnement et faciliter la prise de décision et la négociation. La recherche s'organise autour de la dialectique qui s'instaure entre modèles, expérimentation, observation et instrumentation. La modélisation devient alors un lieu de confrontation d'hypothèses diverses, d'images, de représentations, donc de communication.

Cette problématique est reprise par Gérard Mégie qui insiste non seulement sur la nécessaire interdisciplinarité entre sciences dites exactes, mais par l'indispensable participation des sciences de l'homme et de la société à l'élaboration de stratégies d'ingénierie des territoires. Ce qui donne une place de choix au CNRS, comme l'a prouvé le numéro de La Revue pour l'histoire du CNRS consacré aux recherches sur l'environnement et le dossier d'En petit Comité contenant l'essentiel des débats qui ont eu lieu lors de la organisée par le Comité pour l'histoire du CNRS'1.

Cette thématique est reprise par Paul Caseau qui, après avoir analysé les changements conceptuels dans ce qu'il appelle la boucle " pression-État-réponse ", pose ouvertement une question qui va probablement commander l'avenir : «La société acceptera-t-elle facilement d'entrer dans un processus d'optimisation, c'est-à-dire un processus où le contrôle-commande porte (au moins partiellement) sur ses [de la société] propres comportements? Ne va-t-elle pas réagir en cachant l'information pour sauvegarder ce qu'elle considère comme ses intérêts? » Il est intéressant de constater comment, en effet, la redoutable question de l'environnement a besoin de l'apport des sciences humaines et sociales tout en imposant en contrepartie qu'il y ait des changements de comportement de la part des acteurs sociaux. Or, comme le souligne Claudine SchmidtLainé dans son entretien avec Marcel Jollivet, ce sont d'abord les habitudes des chercheurs qui doivent changer, l'environnement et la modélisation peuvent devenir un lieu de construction de nouvelles problématiques de recherche.

\section{NOTES}

1. En petit Comité, bulletin du Comité pour l'histoire du CNRS, $\mathrm{n}^{\circ} 7$, octobre 2001 : intervention de la table ronde du 13 juin 2001. La Revue pour l'histoire du CNRS, n 4 , mai 2001 : dossier « Environnement ».

\section{INDEX}

Mots-clés : nature, science, sociétés 
AUTEUR

GIROLAMO RAMUNNI

Professeur à l'université de Lyon II 